\title{
Surface Roughness Evolution to Identify Incubation Time for Hot Corrosion of Nickel-Base Superalloys: CMSX-4, CM247LC DS and IN6203DS at $550^{\circ} \mathrm{C}$
}

\author{
Neil Chapman ${ }^{1,2}$ (D) Simon Gray ${ }^{2} \cdot$ Joy Sumner ${ }^{2} \cdot$ John Nicholls $^{2}$
}

Received: 7 June 2020 / Revised: 4 September 2020 / Accepted: 7 September 2020 /

Published online: 24 September 2020

(c) The Author(s) 2020

\begin{abstract}
In the absence of protective scales, nickel-base superalloys have an extremely limited hot corrosion incubation period before increased rates of attack are experienced. This paper reports on the nickel-base superalloys: CMSX-4, CM247LC DS and IN6203DS subjected to $550{ }^{\circ} \mathrm{C}$ hot corrosion exposures of durations ranging from 0 to $800 \mathrm{~h}$, during which none of the superalloys developed a fully protective scale. The aim of the research was to identify the incubation period of each superalloy and this was achieved by means of surface roughness evaluations. A metrology exercise was performed on the cross section of test specimens which produced Cartesian data points which were subsequently converted to Ra and $\mathrm{Rz}$ data. Statistical analysis of the results suggested the incubation period lasted approximately 400, 500 and $200 \mathrm{~h}$, respectively, for each superalloy. It was concluded that refractory metal phases within the microstructure were associated with the relatively short IN6203DS incubation period. This paper demonstrates that monitoring the changes in surface roughness provides a plausible method to identify the transition from incubation to propagation when studying $550{ }^{\circ} \mathrm{C}$ hot corrosion attack.
\end{abstract}

Keywords Liquid sulphate $\cdot$ Solid state $\cdot$ Selective oxidation

\section{Introduction}

A power generating industrial gas turbine (IGT) comprises three sections: compressor, combustion and turbine [1, 2]. The IGT internal components operating downstream of combustion experience both high temperatures and stresses and are

Neil Chapman

chapman.neil@siemens.com

1 Siemens Industrial Turbomachinery Limited, Ruston House, Waterside South, PO Box 1, Lincoln LN5 7FD, UK

2 Cranfield University, College Road, Cranfield, Wharley End, Bedfordshire MK43 0AL, UK 
therefore manufactured from materials, such as nickel-base superalloys, that can endure these conditions. In addition, if corrosive compounds enter the IGT through either the local atmosphere or the fuel used, these materials must be able to cope with the resulting hot corrosion environment.

Hot corrosion can be considered a subset of deposit-induced corrosion [1,2] with the former recognised in aero, marine and IGTs, while the latter is found in many combustion-based power generation systems. Hot corrosion can cause accelerated rates of attack of component alloys [1-4] and as the generic name implies, the presence of deposits such as $\mathrm{Na}_{2} \mathrm{SO}_{4}$ is required to induce the attack [1,2]. The physical condition of the deposits is also an important factor. When deposits accumulate on the components in the molten state, type I hot corrosion occurs [5]. This type of attack is characterised by internal damage/sulphidation and is typically experienced at approximately $900{ }^{\circ} \mathrm{C}$ [6]. An accumulation of deposits in the solid state, providing $\mathrm{SO}_{3}$ is present within the hot gas stream, may also trigger an attack [1, 2]. For this form of hot corrosion, a reaction between the deposits, the $\mathrm{SO}_{3}$ and the oxides of the superalloy may occur which leads to a low melting point deposit forming [5]. For example, it can be $\mathrm{NiO}$ reacting with $\mathrm{SO}_{3}$ to form $\mathrm{NiSO}_{4}$ which further reacts with the $\mathrm{Na}_{2} \mathrm{SO}_{4}$ to form a $\mathrm{Na}_{2} \mathrm{SO}_{4}: \mathrm{NiSO}_{4}$ sulphate system with a lowest melting point of $671{ }^{\circ} \mathrm{C}$ [1]. This gives rise to type II hot corrosion attack experienced at approximately $700{ }^{\circ} \mathrm{C}$ [6] which is typified by pitting [1-8]. Type II attack may also occur after a similar reaction between $\mathrm{CoO}$ and $\mathrm{SO}_{3}$. This forms an even lower melting point sulphate system of $\mathrm{Na}_{2} \mathrm{SO}_{4}: \mathrm{CoSO}_{4}$, which has been cited as reaching $576{ }^{\circ} \mathrm{C}[7], 565{ }^{\circ} \mathrm{C}[1,9]$ or as low as $540{ }^{\circ} \mathrm{C}[4,5]$. For types I and II hot corrosion with a liquid sulphate, a fluxing mechanism dissolves any protective scale from the component surface $[1,2,4,5,7,8,10,11]$, thereby allowing rapid attack of the underlying superalloy as sulphur diffuses inwards [3, 4, 7].

Hot corrosion may also be experienced when deposits remain in the solid state $[8,9,12,13]$. This occurs when the temperatures experienced are below the melting point of the sulphate system that is present [9]. One example of this is given in the literature [9] where hot corrosion exposures produced accelerated attack on the nickel-base superalloy PWA1484 (containing approximately $10 \mathrm{wt} \%$ cobalt) using $\mathrm{Na}_{2} \mathrm{SO}_{4}$ deposits and a gaseous environment of $\mathrm{SO}_{2}$ in oxygen at a temperature of $550{ }^{\circ} \mathrm{C}$. From this example, owing to the various cited melting points of the $\mathrm{Na}_{2} \mathrm{SO}_{4}: \mathrm{CoSO}_{4}$ sulphate system, there could be some debate as to whether the cobalt from the superalloy formed a melted sulphate system or not. However, hot corrosion exposures were also performed under the same conditions on pure nickel (99.98\% purity) which also demonstrated accelerated attack. Since the lowest melting temperature of the $\mathrm{Na}_{2} \mathrm{SO}_{4}: \mathrm{NiSO}_{4}$ sulphate system $\left(671{ }^{\circ} \mathrm{C}\right)$ was much higher than the test temperature $\left(550{ }^{\circ} \mathrm{C}\right)$, the deposits must have remained in the solid state, at least on the pure nickel material. As such, for accelerated attack to happen with a solid sulphate on a nickel-base superalloy, solid-state diffusion must have taken place. Kistler et al. [9] proposed that in such instances, nickel diffuses through porous, fine grained $\mathrm{NiO}$ in order to react with the $\mathrm{Na}_{2} \mathrm{SO}_{4}$. This produces a metastable nanocrystalline $\mathrm{Na}_{2} \mathrm{Ni}_{2} \mathrm{SO}_{5}$-mixed oxide with a structure that allows rapid nickel fluxing into the $\mathrm{Na}_{2} \mathrm{SO}_{4}$. Providing the $\mathrm{Na}_{2} \mathrm{SO}_{4}$ has not sintered, a sustained attack will then be established. After a period of time however, the metastable $\mathrm{Na}_{2} \mathrm{Ni}_{2} \mathrm{SO}_{5}$ 
decomposes back into $\mathrm{NiO}$ and $\mathrm{Na}_{2} \mathrm{SO}_{4}$. This mechanism, summarised for nickel, was also proposed for cobalt, which could form a metastable $\mathrm{Na}_{2} \mathrm{Co}_{2} \mathrm{SO}_{5}$-mixed oxide that eventually decomposes back into $\mathrm{CoO}$ and $\mathrm{Na}_{2} \mathrm{SO}_{4}$ [9].

Hot corrosion has often been described as occurring in two distinct stages [1-10, 13]. The first stage is the incubation period where the rate of attack is low. This is followed by the propagation stage where accelerated attack is experienced. The incubation stage has been defined by various subtly different descriptions including: the time for a liquid melt to form [1], the period where conditions for accelerated attack are established [6] and the period where damage to any protective scale may be repaired by elements within the chemistry of the material $[3,5]$ by selective oxidation [10]. This final definition hints at the nickel-base superalloys' development for growing a protective scale with the purpose of increasing the incubation stage and hence the resistance to hot corrosion attack [1, 2, 4]. That is, additions of aluminium and chromium have been made to the chemistries in order to grow a protective alumina scale (which tends to form at a temperature of $900{ }^{\circ} \mathrm{C}$ and above) or protective chromia scale (which forms below $900{ }^{\circ} \mathrm{C}$ ), respectively [1]. However, under certain circumstances the formation of a protective scale may be hindered. Depending on a superalloy's respective chemistry, refractory metal carbides may be present to varying degrees within the microstructure [14-20]. These types of carbides react with oxygen and therefore have the potential to locally disrupt the formation of the protective scale $[16,17]$. In addition, the carbides may provide shortcircuit diffusion paths either: through the dislocation arrays at the interface with the matrix [21], or by differences in thermal expansion properties [15] which may cause micro-cracking at the interface with the matrix during thermal cycling. Hence, surface-connected refractory metal carbides may shorten or negate the incubation period in localised regions at the surface of the superalloy [16]. In addition, the formation of the protective chromia scale may also be hindered if exposed to an oxidising environment containing $\mathrm{SO}_{2} / \mathrm{SO}_{3}$. In this type of environment, the sulphur may permeate through the chromia scale leaving the superalloy unprotected [1, 22, 23].

In the absence of a protective scale, a question arises as to whether the propagation stage commences immediately or not? Sumner et al. [3] answered this question by analysing metal loss data on the alumina forming nickel-base superalloy CMSX-4 subjected to type II hot corrosion tests. These tests included spraying the tests specimens with a $4 / 1$ molar ratio of $\mathrm{Na}_{2} \mathrm{SO}_{4}: \mathrm{K}_{2} \mathrm{SO}_{4}$ at a flux rate of $5 \mu \mathrm{g} \mathrm{cm}{ }^{-2} \mathrm{~h}^{-1}$ and thermally exposing at a temperature of $700{ }^{\circ} \mathrm{C}$ in an atmosphere of $300 \mathrm{ppm} \mathrm{SO}$ in air. The results indicated that the CMSX-4 superalloy entered the propagation stage after approximately $40 \mathrm{~h} \mathrm{[3].} \mathrm{The} \mathrm{deposits} \mathrm{used,} \mathrm{however,} \mathrm{had} \mathrm{a} \mathrm{lowest} \mathrm{melting} \mathrm{point}$ of $831{ }^{\circ} \mathrm{C}$ [1] and, considering the test temperature, it was plausible that during this time, $\mathrm{NiO}$ and $\mathrm{CoO}$ was generated and reacted with the $\mathrm{SO}_{3}$ to form $\mathrm{NiSO}_{4}$ and $\mathrm{CoSO}_{4}$, respectively, both of which further reacted with the $\mathrm{Na}_{2} \mathrm{SO}_{4}: \mathrm{K}_{2} \mathrm{SO}_{4}$ to produce a sulphate system that was in the melted state at the test temperature. For hot corrosion tests performed below the melting point of the sulphate system present, the duration of the incubation period may be assumed to be the time required to form metastable nanocrystalline-mixed oxides.

Due to the importance of incubation periods and the potential link to the development of deposit chemistries, this research aims to investigate the relative duration 
of the incubation periods of three nickel-base superalloys: CMSX-4 (classed as an alumina former), CM247LC DS (also classed as an alumina former) and IN6203DS (classed as a chromia former). In particular, the research focusses on hot corrosion exposures carried out at $550{ }^{\circ} \mathrm{C}$, as at this temperature, the hot corrosion may attack the superalloys by solid-state diffusion before any slow growing protective scale forms a continuous layer. Hot corrosion progression was tracked, and links made to the evolution of surface roughness (due to non-uniform metal loss) over time on all three superalloys. This allowed an assessment of the extent of the respective incubation periods.

\section{Materials and Methods}

\section{Materials and Test Specimens}

The investigation was performed on three nickel-base superalloys which included the alumina formers CMSX-4 and CM247LC DS along with the chromia former IN6203DS. The composition of these three superalloys are shown in Table 1 and the weight percentages were obtained from the respective materials certificates. The measured values quoted were obtained using the X-ray fluorescence method except for carbon (obtained using LECO analysis) and the base element, nickel, which has been arithmetically calculated.

Each superalloy had been cast in the $<001>$ orientation in the form of bars which were single point turned to a diameter of $6 \mathrm{~mm}$. The machined bars were then sectioned to obtain test specimens which were $10 \mathrm{~mm}$ in length.

\section{Hot Corrosion Tests}

The test specimens were cleaned in isopropyl alcohol before dimensional data (using a calibrated gauge with a resolution of $1 \mu \mathrm{m}$ and accuracy of $2 \mu \mathrm{m}$ ) and room temperature weight data (using calibrated scales with a resolution of $0.01 \mathrm{mg}$ ) were obtained. Next, the test specimens were placed on a hot plate and heated to a temperature of $180{ }^{\circ} \mathrm{C}$. Immediately after this, a 4/1 molar ratio of $\mathrm{Na}_{2} \mathrm{SO}_{4}: \mathrm{K}_{2} \mathrm{SO}_{4}$ salt deposits, dissolved in water, was sprayed as evenly as possible around the outer surfaces of the test specimens with a nominal surface loading of $500 \mu \mathrm{g} \mathrm{cm}^{-2}$. On contact with the hot surfaces of the test specimens, the water flash evaporated leaving the salts adhering to the surfaces. The test specimens were then reweighed, on

Table 1 Composition of the superalloys in wt $\%$

\begin{tabular}{llllllllllllll}
\hline Superalloy & $\mathrm{Ni}$ & $\mathrm{C}$ & $\mathrm{Cr}$ & $\mathrm{Co}$ & $\mathrm{W}$ & $\mathrm{Nb}$ & $\mathrm{Ta}$ & $\mathrm{Hf}$ & $\mathrm{Ti}$ & $\mathrm{Al}$ & $\mathrm{Re}$ & $\mathrm{Zr}$ & $\mathrm{Mo}$ \\
\hline CMSX-4 & 61.2 & 0.004 & 6.5 & 9.5 & 6.4 & - & 6.4 & 0.1 & 1.0 & 5.5 & 2.8 & - & 0.6 \\
CM247LC DS & 61.5 & 0.08 & 8.1 & 9.3 & 9.5 & - & 3.2 & 1.5 & 0.7 & 5.6 & - & 0.01 & 0.5 \\
IN6203DS & 48.8 & 0.16 & 21.4 & 18.7 & 2.0 & 0.8 & 1.1 & 1.1 & 3.5 & 2.3 & - & 0.06 & $<0.1$ \\
\hline
\end{tabular}


the calibrated scales, at room temperature. The dimensional and room temperature weight data were used to confirm the nominal surface loading had been achieved for each specimen.

The salted test specimens were then thermally exposed at a temperature of $550{ }^{\circ} \mathrm{C}$ $\left( \pm 5^{\circ} \mathrm{C}\right)$ in a horizontal tube furnace containing an atmosphere of $300 \mathrm{ppm} \mathrm{SO}_{2}$ in air that had a flow rate of 50 SCCM. The gas was vented through bubblers using a sodium hydroxide solution as a scrubber. The thermal exposure was interrupted every $100 \mathrm{~h}$ to allow the test specimens to cool to room temperature. During each interruption, a randomly selected test specimen from each material was removed from the test. The remaining specimens had the salts re-applied using the same technique as previously described before the hot corrosion exposures recommenced. This methodology was repeated until each superalloy had one test specimen exposed to the $550{ }^{\circ} \mathrm{C}$ hot corrosive environment for each duration of $100,200,300,400$, $500,600,700$ and $800 \mathrm{~h}$.

\section{Surface Roughness Data Gathering}

Following exposure to the hot corrosion tests, the specimens were mounted in resin and the cross sections polished to a $1 \mu \mathrm{m}$ finish. After this, each mounted specimen was subjected to a metrology exercise using an optical microscope in conjunction with calibrated bespoke measuring software. This allowed collection of surface roughness data, where the surface was defined by the interface between the corrosion scale and the good metal. Twelve 300- $\mu$ m-long equispaced locations around the perimeter of the test specimen's cross section were used to collect the surface roughness data by obtaining 28 roughly equispaced Cartesian data points from each location. The Cartesian data points were used to calculate a centre point of the cross section of the test specimen which then allowed further calculations to derive radii data.

For each location, the radii were used to produce ordinate data by subtracting the mean radii value from each of the calculated radii. The ordinate values were subsequently used to evaluate the $\mathrm{Ra}$ and $\mathrm{Rz}$ surface roughness as detailed in BS1134:2010 [24]—-see Theory/calculation section of this paper. Hence, the surface roughness on the cross section of the test specimen at the interface between the corrosion scale and the good metal was defined by $12 \mathrm{Ra}$ and $\mathrm{Rz}$ datasets as determined by the 28 measurement points at each measurement location.

The surface roughness data gathering methodology was also repeated on an asmachined specimen from each superalloy in order to obtain reference data.

\section{Surface Roughness Data Analysis}

Analysis of the data for each superalloy was performed using boxplots, one-way ANOVA (analysis of variance) tests and scatter plots in conjunction with the statistical software: Minitab version 18 [25].

Boxplots are a graphical representation of datasets (in this case: $\mathrm{Ra}$ and $\mathrm{Rz}$ ) and show the median, the lower and upper quartiles, and the range. Minitab also identifies any unusual data points within the respective dataset and highlights these as 
outliers, reported in the form of an asterisk on the boxplot. An outlier is defined by Minitab as any data point which is greater or lower than the upper or lower quartile data point, respectively, by a factor of 1.5 of the interquartile range [25]. All outliers identified were investigated further to ensure errors had not occurred during the data gathering, calculation of the $\mathrm{Ra}$ and $\mathrm{Rz}$ values, or data input into Minitab. If the outliers were error free and considered genuine data, the respective values were retained within the respective dataset. Displaying the boxplots for each superalloy in order of the duration of exposure to the $550^{\circ} \mathrm{C}$ hot corrosion conditions $(0,100$, $200,300,400,500,600,700$ and $800 \mathrm{~h}$ ) therefore allowed a visual assessment of the evolution of the surface roughness.

The one-way ANOVA test was selected, along with a 0.05 significance level, to test the null hypothesis that the Ra or Rz datasets of each superalloy had plausibly the same mean value. The alternative hypothesis was that at least one dataset had a different mean value [25]. Minitab calculates a probability ( $P$ value) for the collected data to have occurred which is subsequently compared with the significance level. $P$ values less than or equal to the significance level result in rejection of the null hypothesis in favour of the alternative hypothesis. $P$ values greater than the significance level result in the null hypothesis not being rejected and therefore being adopted [25]. Since the dataset for the as-machined specimen was included in the testing, the one-way ANOVA tests allowed an evaluation of the duration wherein the surface roughnesses of exposed test specimens were plausibly the same as that of the as-machined surface. This duration was considered the incubation period of the $550{ }^{\circ} \mathrm{C}$ hot corrosion. Once a dataset was highlighted as having an increased mean value, in $\mathrm{Ra}$ or $\mathrm{Rz}$, when compared with the as-machined specimen, the superalloy was considered to have entered the propagation stage.

Assumptions for the one-way ANOVA test include that the datasets being compared are normally distributed and have constant variance. These assumptions may be checked visually from the symmetry and length of the boxplots along with intest checks whilst using Minitab. It is confirmed that all the results of the one-way ANOVA tests quoted within this paper were based on datasets that were plausibly normally distributed with constant variance.

Extracting a suitable extreme value from the Rz datasets also allowed an approximation of the maximum penetration rate of the $550{ }^{\circ} \mathrm{C}$ hot corrosion attack as a function of exposure time. To avoid any outlier becoming overly influential in this approximation, the maximum $\mathrm{Rz}$ values were not selected for this purpose. Instead, the approximation of the maximum penetration rate was based on the upper quartile data point. By plotting these data points in the form of a scatter plot, trend lines (based on the results of the one-way ANOVA tests) could be inserted and used to further define the duration of the incubation stage of $550{ }^{\circ} \mathrm{C}$ hot corrosion for the three superalloys under investigation.

\section{SEM/EDX Analysis}

Energy-dispersive X-ray (EDX) mapping, with an accelerating voltage of $20 \mathrm{kV}$, was performed in conjunction with back-scattered electron imaging using a JEOL 
JSM-6460 SEM and Inca software [26]. Analysis was carried out on the corrosive scales on the specimens of each superalloy that had completed $800 \mathrm{~h}$ of exposure to the test conditions. This was used to confirm whether a protective scale had, or had not, formed on the superalloys during exposure to the $550{ }^{\circ} \mathrm{C}$ hot corrosion conditions.

\section{Theory/Calculation}

The Ra and Rz definitions are described within BS1134:2010 [24]. These definitions are briefly summarised within this section of the paper.

The Ra measurement is an arithmetic mean of the absolute ordinate values within the sampling length. This is calculated as per Eq. 1:

$$
\mathrm{Ra}=\frac{1}{n} \sum_{i=1}^{n}\left|Z_{i}\right|
$$

where $n$ the number of measurements in the sampling length, $Z_{i}$ the ordinate values within the sampling length and $i$ the measurement point number.

The Rz measurement (Eq. 2) is the sum of the magnitudes of the largest peak and valley within the sampling length.

$$
\mathrm{Rz}=\left|Z_{\max }\right|+\left|Z_{\min }\right|
$$

where $\left|Z_{\max }\right|$ magnitude of the largest peak and $\left|Z_{\min }\right|$ magnitude of the largest valley.

\section{Results and Discussion}

Each superalloy under investigation, CMSX-4, CM247LC DS and IN6203DS, experienced non-uniform metal loss giving rise to an increase in surface roughness (Fig. 1) during the $800 \mathrm{~h}$ of exposure to the $550{ }^{\circ} \mathrm{C}$ hot corrosion conditions. Figure 2 illustrates the evolution of the $\mathrm{Ra}$ and $\mathrm{Rz}$ surface roughness during this period in the form of boxplots. Visual assessments of the boxplots revealed a similar pattern for both the $\mathrm{Ra}$ and $\mathrm{Rz}$ data from each superalloy. That is, the Ra and $\mathrm{Rz}$ surface roughness initially appeared to show an extremely subtle positive trend with exposure time, albeit with significant overlap of the data with that of the as-machined specimen ( $0 \mathrm{~h}$ exposure). After the initial period, both the $\mathrm{Ra}$ and $\mathrm{Rz}$ data experienced an increase in the rate of the surface roughening over time, with the respective boxplots suggesting this may have occurred after approximately: $400 \mathrm{~h}$ for CMSX-4, $500 \mathrm{~h}$ for CM247LC DS and $200 \mathrm{~h}$ for IN6203DS.

The respective boxplots for each superalloy also showed that during the initial, extremely subtle, increase in the surface roughening, the $\mathrm{Ra}$ and $\mathrm{Rz}$ datasets were plausibly normally distributed with constant variance. Once the superalloys experienced the increase in rate of the surface roughening, the datasets still appeared 

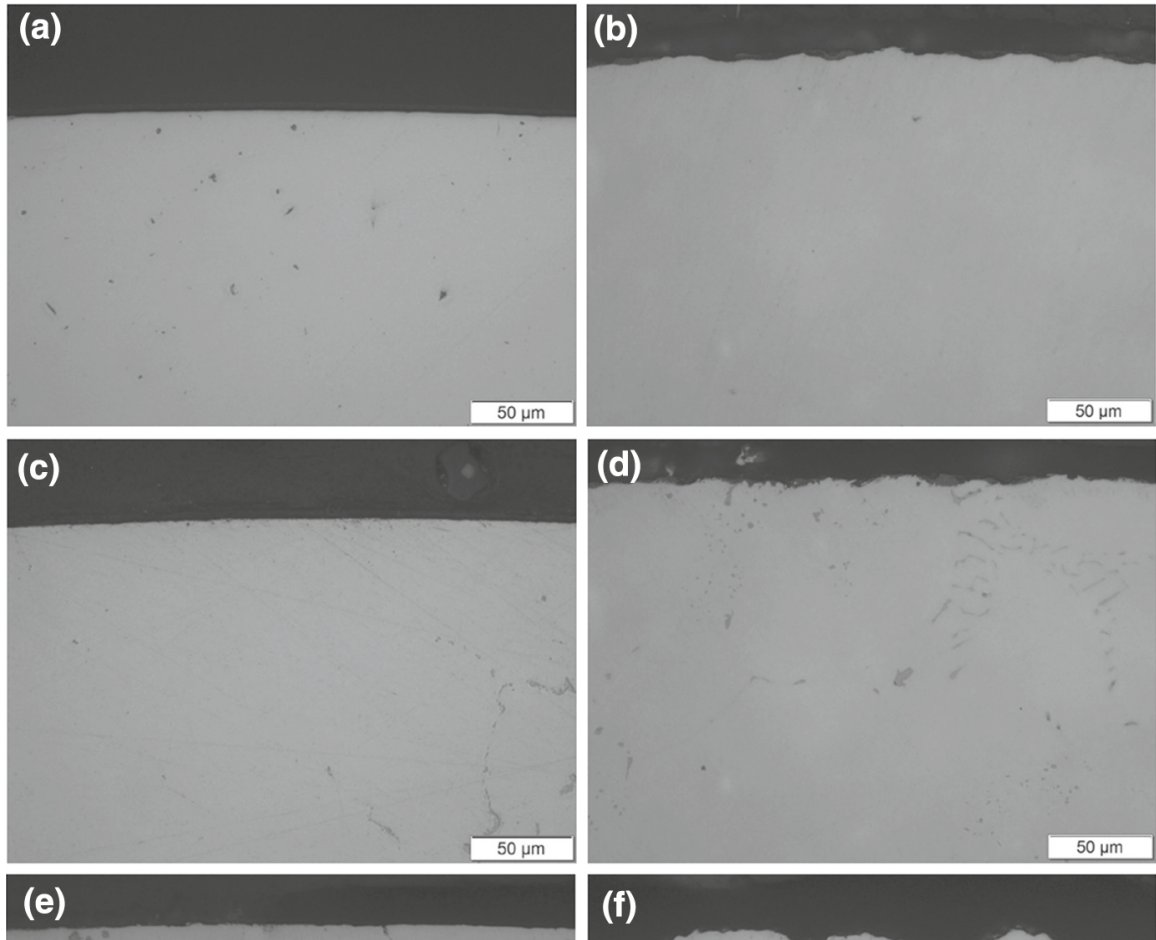

Fig. 1 Cross sections of superalloys showing surface roughness comparisons before and after exposure to the tested $550{ }^{\circ} \mathrm{C}$ hot corrosion conditions. Images include CMSX-4 before (a) and after $800 \mathrm{~h}$ exposure (b), CM247LC DS before (c) and after $800 \mathrm{~h}$ exposure (d), and IN6203DS before (e) and after $800 \mathrm{~h}$ exposure (f)

to be normally distributed but generally with increasing variance as the exposure duration increased. These observations confirmed the validity of performing the one-way ANOVA tests for the purpose of providing statistical evidence supporting the boxplot visual assessment. That is, the one-way ANOVA tests were performed to determine the first dataset which was not plausibly the same as that of the as-machined specimen. The $\mathrm{Ra}$ and $\mathrm{Rz}$ results of the one-way ANOVA tests (Table 2) agreed for each superalloy. Specifically, the Ra and Rz results suggested the first CMSX-4 dataset which was different from that of the as-machined specimen was the $500 \mathrm{~h}$ dataset. A similar analysis of the CM247LC DS data 


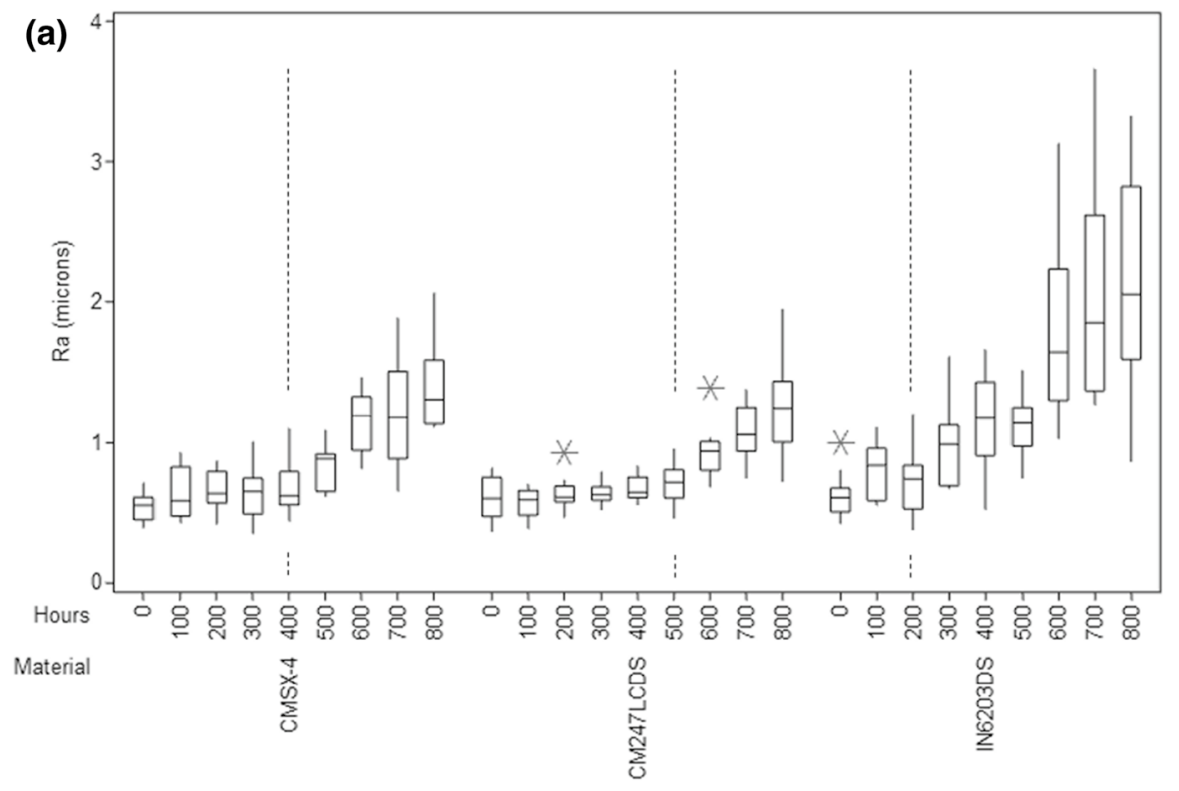

(b)

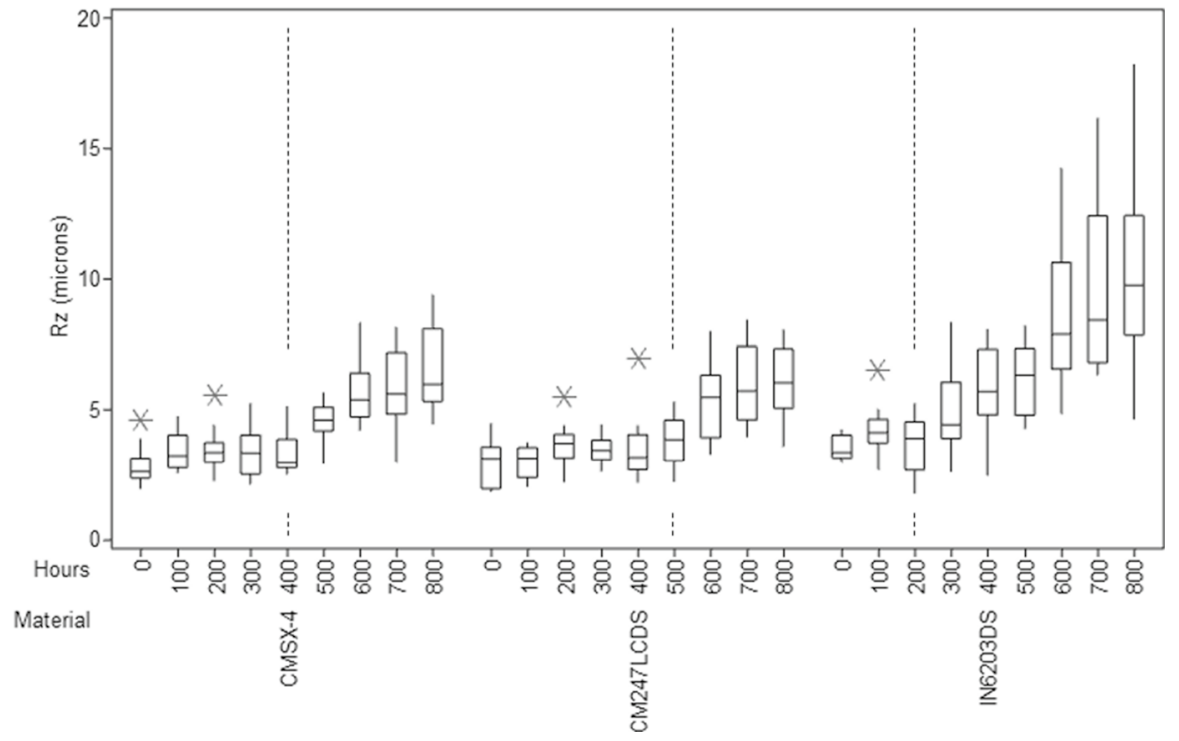

Fig. 2 Illustration of the evolution of the (a) Ra and (b) Rz surface roughness with respect to exposure to the tested $550{ }^{\circ} \mathrm{C}$ hot corrosion conditions. The vertical dashed lines represent the approximate duration when the transition of hot corrosion from incubation to propagation occurs. The asterisks denote the outliers within the respective datasets 
Table 2 One-way ANOVA results of $\mathrm{Ra}$ and $\mathrm{Rz}$ datasets

\begin{tabular}{lllll}
\hline Superalloy & Analysis & Data sets (h) & $P$ value & $\begin{array}{l}\text { Reject the null hypothesis } \\
\text { in favour of the alterna- } \\
\text { tive? }\end{array}$ \\
\hline CMSX-4 & Ra & $0,100,200,300$ and 400 & 0.289 & No \\
CMSX-4 & Rz & $0,100,200,300$ and 400 & 0.315 & No \\
CMSX-4 & Ra & $0,100,200,300,400$ and 500 & 0.003 & Yes \\
CMSX-4 & $\mathrm{Rz}$ & $0,100,200,300,400$ and 500 & 0.000 & Yes \\
CM247LC DS & $\mathrm{Ra}$ & $0,100,200,300,400$ and 500 & 0.060 & No \\
CM247LC DS & $\mathrm{Rz}$ & $0,100,200,300,400$ and 500 & 0.194 & No \\
CM247LC DS & $\mathrm{Ra}$ & $0,100,200,300,400,500$ and 600 & 0.000 & Yes \\
CM247LC DS & $\mathrm{Rz}$ & $0,100,200,300,400,500$ and 600 & 0.000 & Yes \\
IN6203DS & $\mathrm{Ra}$ & 0,100 and 200 & 0.085 & No \\
IN6203DS & $\mathrm{Rz}$ & 0,100 and 200 & 0.117 & No \\
IN6203DS & $\mathrm{Ra}$ & $0,100,200$ and 300 & 0.004 & Yes \\
IN6203DS & $\mathrm{Rz}$ & $0,100,200$ and 300 & 0.011 & Yes \\
\hline
\end{tabular}

indicated that the first dataset for this superalloy that was different to that of the as-machined specimen was the $600 \mathrm{~h}$ dataset. The analysis of the IN6203DS data suggested the $300 \mathrm{~h}$ dataset was the first that was plausibly different from the asmachined specimen.

The boxplots and one-way ANOVA tests have indicated that all three superalloys experienced an increase in the rate of surface roughening during exposure to the $550{ }^{\circ} \mathrm{C}$ hot corrosion conditions. These increases appeared to be consistent with a transition from the incubation stage to the propagation stage of the $550{ }^{\circ} \mathrm{C}$ hot corrosion. To determine more precisely the time to transition, the $\mathrm{Rz}$ upper quartile data points were split into two data series for each superalloy and plotted against exposure time (Fig. 3). The split in the data was based on the last exposure duration where the surface roughness was plausibly the same as that of the as-machined specimen (and this dataset was included in both data series). Trend line equations were then used to estimate the point of transition for each superalloy by determining simultaneous solutions. These calculations estimated the 'actual' duration of the incubation stage before the transition to propagation as: $404 \mathrm{~h}$ for CMSX-4, $415 \mathrm{~h}$ for CM247LC DS and $220 \mathrm{~h}$ for IN6203DS. The results of these calculations are consistent with the results of the boxplots and one-way ANOVA tests for the CMSX-4 and IN6203DS superalloys but not for CM247LC DS. The lack of consistency for CM247LC DS appears to be the result of the $800 \mathrm{~h} \mathrm{Rz}$ dataset for this superalloy, underestimating the true surface roughness (Figs. 2, 3). This may have occurred due to the data being obtained from 12 locations, each with arc lengths of $300 \mu \mathrm{m}$, which represented approximately $19 \%$ of the total perimeter of the cross section of the test specimen, missing the worst of the surface roughness. This would have meant that the $800 \mathrm{~h} \mathrm{Rz}$ upper quartile data point acted in an overly influential manner resulting in the underestimation of the point of transition from the incubation to propagation stage for CM247LC DS. 
The equations describing the trend lines within Fig. 3 also provided an estimation of the maximum penetration rate of the $550{ }^{\circ} \mathrm{C}$ hot corrosion attack during the incubation and propagation stages. For both stages, the rate was greatest for IN6203DS which provided further confirmation that this superalloy had the least resistance to the $550{ }^{\circ} \mathrm{C}$ hot corrosion conditions. This resulted in IN6203DS suffering greater levels and variance of surface roughening (Fig. 2) than the CMSX-4 and CM247LC DS superalloys at the end of the testing.

To investigate why IN6203DS had the lowest hot corrosion resistance to the exposure conditions, EDX mapping using an accelerating voltage of $20 \mathrm{kV}$ in conjunction with back-scattered electron imaging was performed on the scale of each superalloy sample after exposure for $800 \mathrm{~h}$. EDX analysis though is unable to differentiate between molybdenum, which all three superalloys contained small quantities of (Table 1), and sulphur. This is due to these elements possessing overlapping $\mathrm{X}$-ray energy peaks. However, as the salt deposits were sprayed onto the surfaces of the test specimens, it was expected that a sulphur content would exist within the scales. Therefore, any sulphur/molybdenum indication was assumed to be sulphur.

The EDX mapping performed on the CMSX-4 specimen (Fig. 4) indicated an external surface consisting mainly of nickel, cobalt, sulphur and oxygen suggesting sulphates formed. Underneath this, an aluminium/chromium oxide had formed which appeared to be non-protective as evidenced by the sodium and sulphur levels within the oxide.

A similar EDX map of the CM247LC DS superalloy (Fig. 5) led to similar conclusions as for the CMSX-4 superalloy with the exception that the external surface appeared to be mainly nickel and sulphur. Although oxygen did not appear to be associated with these elements, it was thought that these were still sulphates based on the test temperature.

The EDX mapping of IN6203DS (Fig. 6) indicated that a protective chromia scale had not formed on this superalloy. Instead, a complex scale involving the elements: oxygen, aluminium, chromium, cobalt, nickel, titanium, hafnium, tantalum and zirconium appeared to be present. This complex scale also provided little protection against the $550{ }^{\circ} \mathrm{C}$ hot corrosion as evidenced by the sodium and sulphur within the scale leaving IN6203DS prone to surface roughening. In addition, the EDX mapping revealed phases within the IN6203DS microstructure which contained the refractory elements: titanium, hafnium, tantalum and zirconium. These phases were most likely carbides and the surface roughening appeared to be at its greatest in the material adjacent to these carbides (Fig. 6). SEM investigations of similar refractory metal carbides that were remote from the surface (Fig. 7) suggested a mismatch in the lattice structure with the matrix. This was possibly caused by the thermal cycling during the $550{ }^{\circ} \mathrm{C}$ hot corrosion exposure creating micro-cracking around the carbides owing to a difference in the thermal expansion coefficients as compared to the matrix, or may have been due to sample preparation. It is therefore proposed that for refractory metal carbides connected to the surface, the respective interfaces with the matrix acted as shortcircuit diffusion paths. This was most likely due to the dislocation arrays which exist at these locations, or possibly the potential thermal cycle micro-cracking, which enabled sulphur to diffuse into and combine with the sulphur attack from 

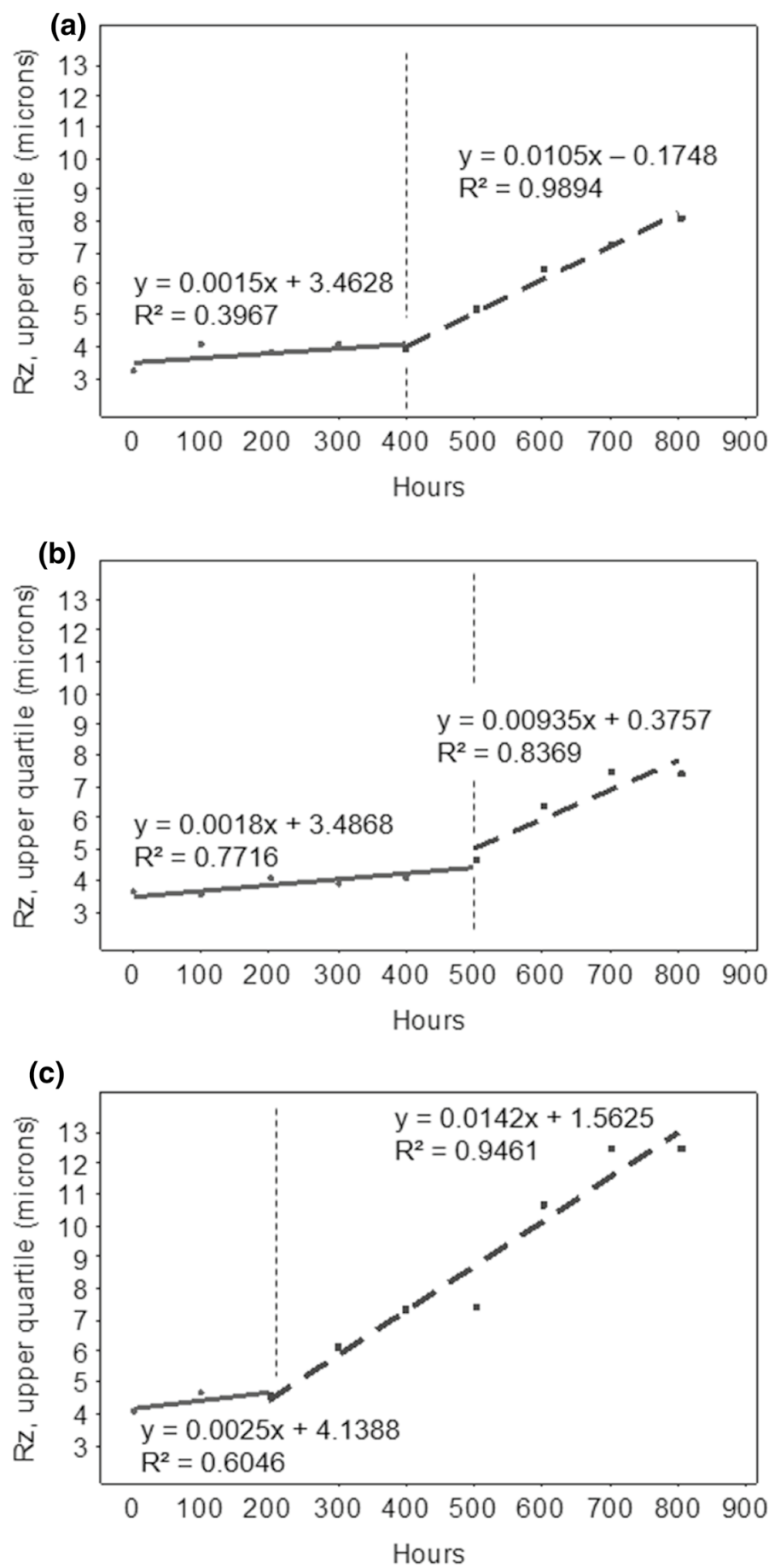
4 Fig. 3 Illustration of the Rz upper quartile data points, split into two series approximating the maximum $550{ }^{\circ} \mathrm{C}$ hot corrosion penetration rates during the incubation and propagation stages in (a) CMSX-4, (b) CM247LC DS and (c) IN6203DS superalloys. The vertical dashed lines represent the approximate duration when the transition of hot corrosion from incubation to propagation occurs
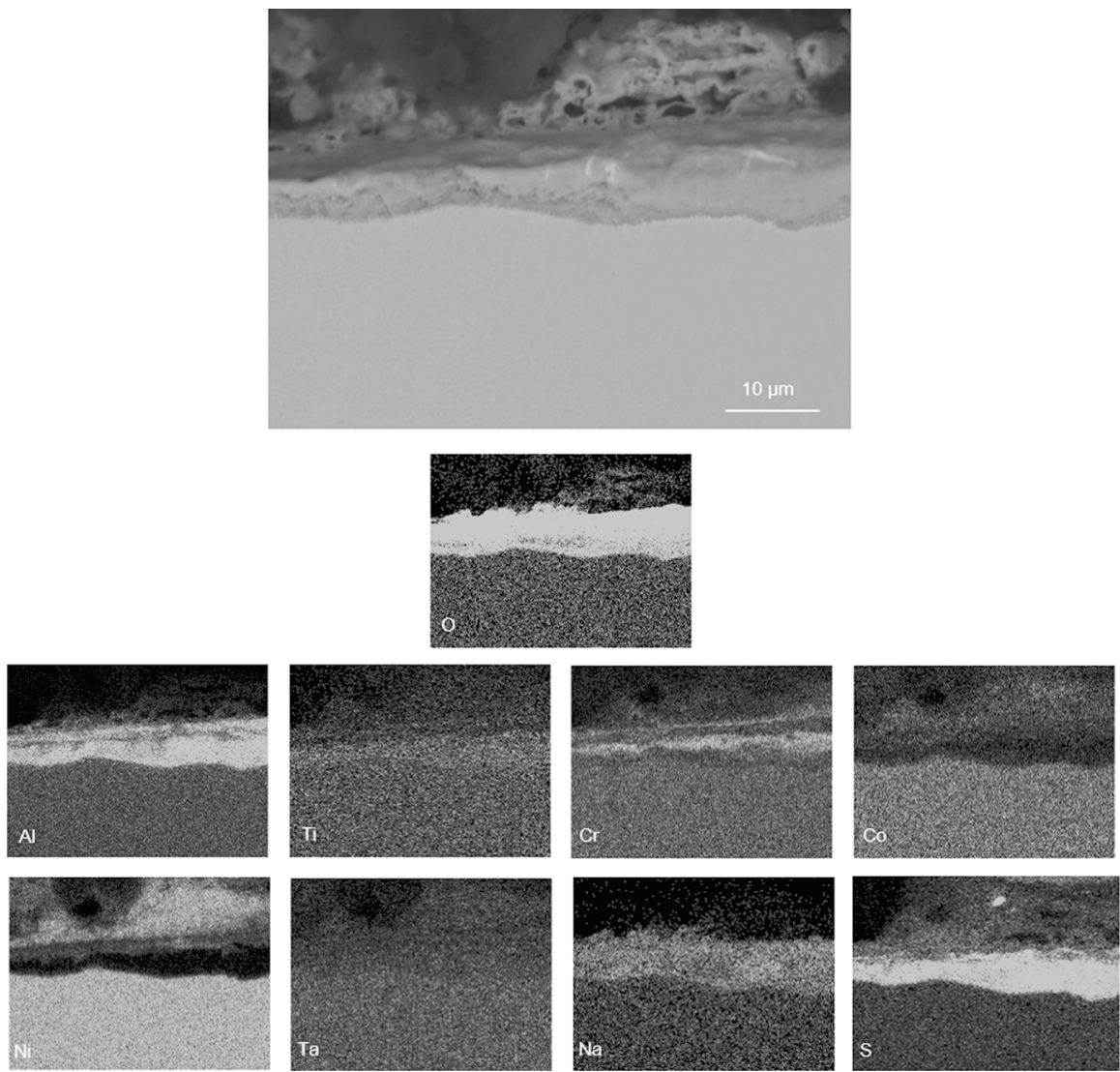

Fig. 4 Back-scattered electron images and EDX elemental maps of the scale on CMSX-4 superalloy after $800 \mathrm{~h}$ exposure to the tested $550{ }^{\circ} \mathrm{C}$ hot corrosion

the surface causing surface roughening adjacent to the carbides. Based on this, it is highly plausible that the refractory metal carbides were responsible for IN6203DS showing the lowest resistance to the exposure conditions and experiencing greater levels and variance of surface roughening. 

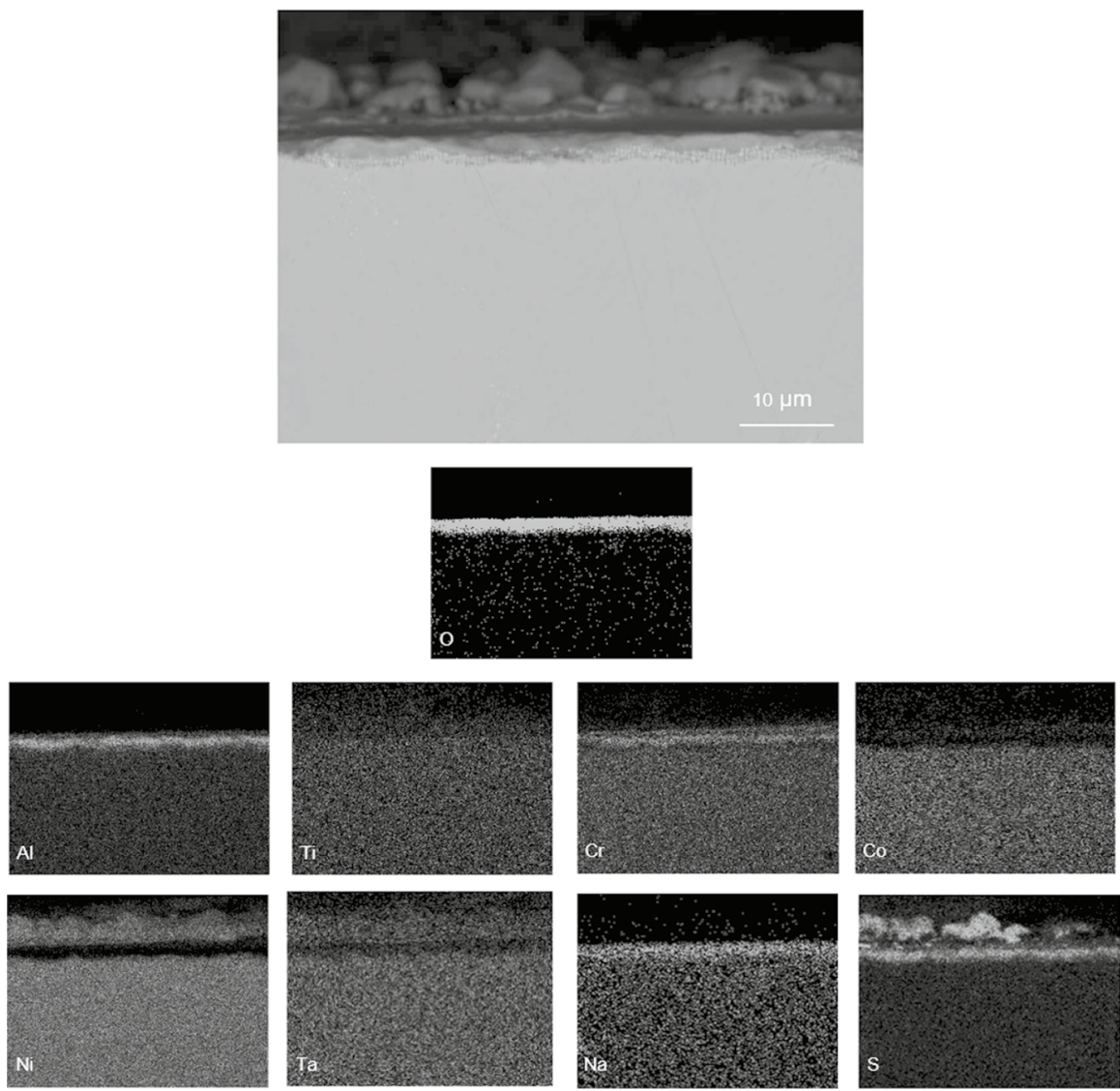

Fig. 5 Back-scattered electron images and EDX elemental maps of the scale on CM247LC DS superalloy after $800 \mathrm{~h}$ exposure to the tested $550{ }^{\circ} \mathrm{C}$ hot corrosion conditions

\section{Conclusions}

- During exposure to the $550{ }^{\circ} \mathrm{C}$ hot corrosion conditions, none of the superalloys tested developed a protective scale. In the case of the alumina formers, CMSX-4 and CM247LC DS, this was due to the test temperature. At $550{ }^{\circ} \mathrm{C}$, the slow growth of transient aluminas meant that a protective scale could not form, and only transient oxides of all alloying additions formed. In the case of the chromia former, IN6203DS, the refractory metal carbides within the microstructure disrupted the formation of the protective scale thus allowing $\mathrm{SO}_{2}$ within the gas phase direct access to the substrate.

- All three superalloys experienced surface roughening during the $550{ }^{\circ} \mathrm{C}$ hot corrosion tests. Initially, the surface roughening was very slight, and this plausibly gave the same $\mathrm{Ra}$ and $\mathrm{Rz}$ values as that of an as-machined surface. After 

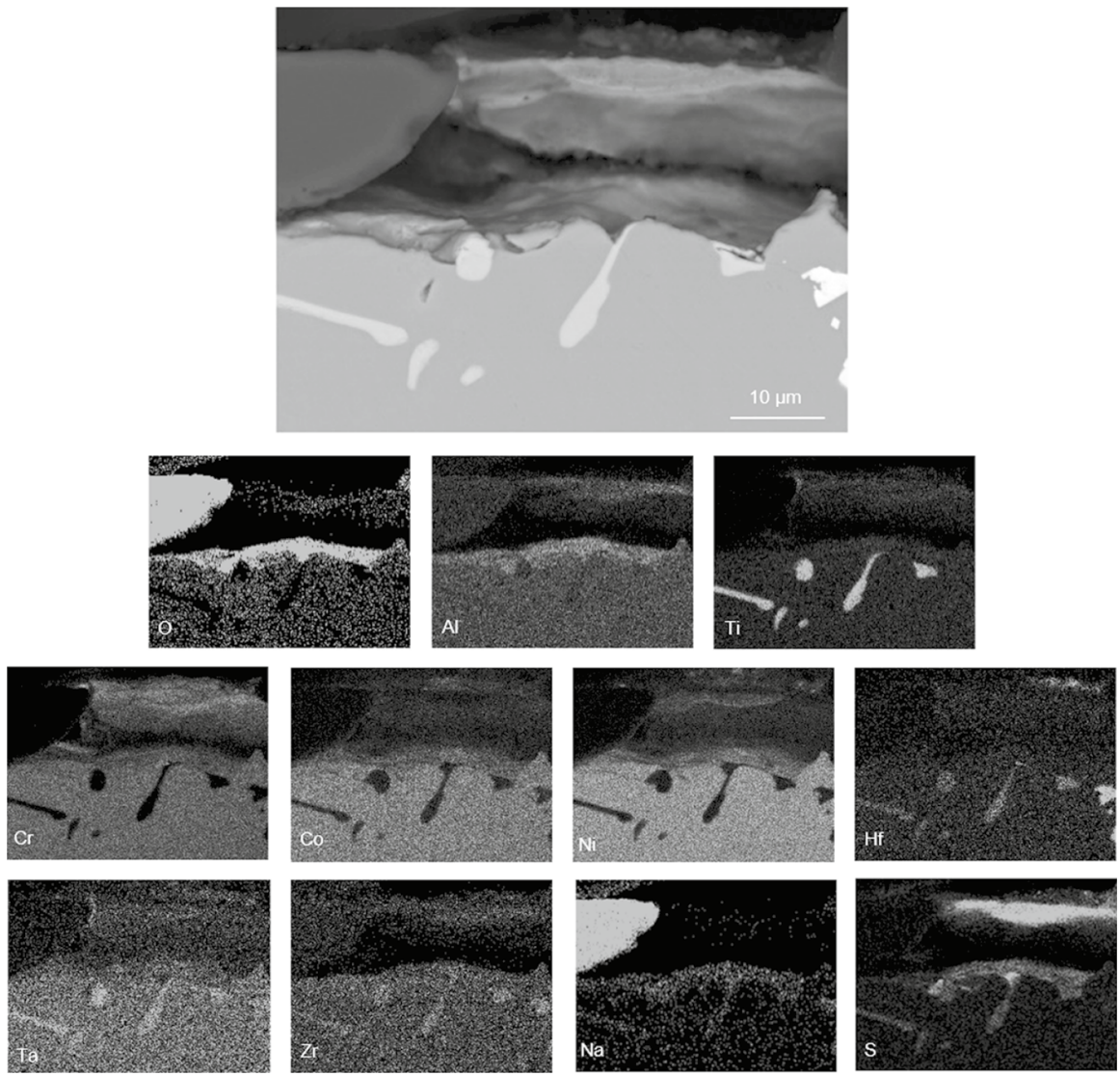

Fig. 6 Back-scattered electron images and EDX elemental maps of the scale on IN6203DS superalloy after $800 \mathrm{~h}$ exposure to the tested $550{ }^{\circ} \mathrm{C}$ hot corrosion conditions

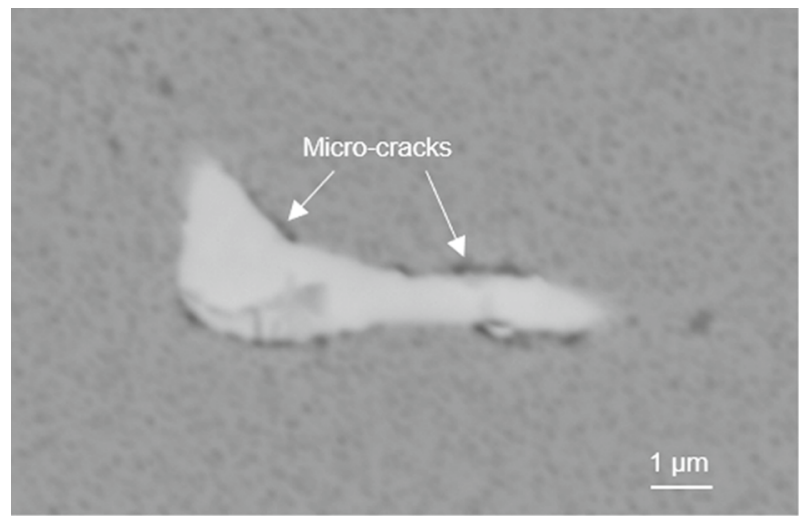

Fig. 7 Refractory metal carbide phase within IN6203DS superalloy showing micro-cracking at interface with the matrix 
a prolonged exposure period though, the rate of surface roughening increased, and this was the point where the superalloys were considered to have transitioned from the incubation stage to the propagation stage of hot corrosion. This occurred after approximately $400 \mathrm{~h}$ for CMSX-4, $500 \mathrm{~h}$ for CM247LC DS and $200 \mathrm{~h}$ for IN6203DS.

- A boxplot comparison of the Ra and Rz values, along with scatter plots of the $\mathrm{Rz}$ upper quartile data points, indicated IN6203DS had suffered greater levels and variance of surface roughening than either CMSX-4 or CM247LC DS. This was attributed to the significantly greater refractory metal carbide content within the IN6203DS superalloy. It was proposed that for surface-connected carbides, the interface with the matrix acted as short-circuit diffusion paths. This was most likely due to the associated dislocation arrays or possibly any micro-cracking that occurred during thermal cycling owing to a difference in the thermal expansion coefficients between the carbides and the matrix. The short-circuit diffusion paths allowed sulphur to diffuse along the metal/scale interface exacerbating the hot corrosion attack from the surface, thereby causing enhanced surface roughening.

Acknowledgements The authors would like to thank Siemens Industrial Turbomachinery Limited for funding this work.

Author Contributions All authors contributed to the conception and design of the study. Material preparation, experimental work, data collection/analysis and imaging were performed by NC. The first draft of the manuscript was written by NC. All authors commented on previous versions of the manuscript. All authors read and approved the final manuscript.

Funding This research has been funded by Siemens Industrial Turbomachinery Limited.

Availability of Data and Material The data cannot be shared at this time as it is part of an ongoing study.

\section{Compliance with Ethical Standards}

Conflict of interest The authors declare that they have no conflict of interest.

Open Access This article is licensed under a Creative Commons Attribution 4.0 International License, which permits use, sharing, adaptation, distribution and reproduction in any medium or format, as long as you give appropriate credit to the original author(s) and the source, provide a link to the Creative Commons licence, and indicate if changes were made. The images or other third party material in this article are included in the article's Creative Commons licence, unless indicated otherwise in a credit line to the material. If material is not included in the article's Creative Commons licence and your intended use is not permitted by statutory regulation or exceeds the permitted use, you will need to obtain permission directly from the copyright holder. To view a copy of this licence, visit http://creativecommons.org/licen ses/by/4.0/.

\section{References}

1. B. Bourdenet, High Temperature Corrosion in Gas Turbines: Thermodynamic Modelling and Experimental Results [PhD thesis]. Saarbrucken: RWTH Aachen University (2004). 
2. H. Lai, High Temperature Oxidation and Corrosion of Ni-Based Superalloys for Industrial Gas Turbines [PhD thesis]. Goteborg: Chalmers University of Technology (2014).

3. J. Sumner, A. Encinas-Oropesa, N. J. Simms and J. R. Nicholls, Oxidation of Metals 80, 2013 (553). https://doi.org/10.1007/s11085-013-9395-x.

4. N. Eliaz, G. Shemesh and R. M. Latanision, Engineering Failure Analysis 9, 2002 (31). https:// doi.org/10.1016/S1350-6307(00)00035-2.

5. S. Kalsi, International Journal on Emerging Technologies 7, 2016 (133).

6. J. Sumner, A. Encinas-Oropesa, N. J. Simms and J. R. Nicholls, Materials and Corrosion 65, 2014 (188). https://doi.org/10.1002/maco.201307425.

7. P. Lortrakul, R. W. Trice, K. P. Trumble and M. A. Dayananda, Corrosion Science 80, 2014 (408). https://doi.org/10.1016/j.corsci.2013.11.048.

8. F. Pettit, Oxidation of Metals 76, 2011 (1). https://doi.org/10.1007/s11085-011-9254-6.

9. E. Kistler, W. T. Chen, G. H. Meier and B. Gleeson, Materials and Corrosion 70, 2019 (1346). https://doi.org/10.1002/maco.201810751.

10. N. Birks, G. H. Meier and F. S. Petit, Journal of Metals 39, 1987 (28). https://doi.org/10.1007/ BF03257568.

11. R. A. Rapp, Corrosion Science 44, 2002 (209). https://doi.org/10.1016/S0010-938X(01)00057-9.

12. W. J. Zhang and R. Sharghi-Moshtaghin, Metallurgical and Materials Transactions A 49, 2018 (4362). https://doi.org/10.1007/s11661-018-4755-4.

13. J. M. Alvarado-Orozco, J. E. Garcia-Herrera, B. Gleeson, F. S. Petit and G. H. Meier, Oxidation of Metals 90, 2018 (527). https://doi.org/10.1007/s11085-018-9853-6.

14. R. C. Reed, The Superalloys Fundamentals and Applications, (Cambridge University Press, Cambridge, 2006), p. 35.

15. M. J. Donachie and S. J. Donachie, Superalloys a Technical Guide, 2nd ed, (ASM International, $\mathrm{OH}, 2003)$, p. 27.

16. A. Potter, J. Sumner and N. J. Simms, Materials at High Temperature 35, 2017 (236). https://doi. org/10.1080/09603409.2017.1392413.

17. D. Gozzi, G. Guzzardi, M. Montozzi and P. L. Cignini, Solid State Ionics 101-103, 1997 (1243). https://doi.org/10.1016/S0167-2738(97)00413-X.

18. N. S. Husseini, D. P. Kumah, J. Z. Yi, et al., Acta Materialia 56, 2008 (4715). https://doi. org/10.1016/j.actamat.2008.05.041.

19. P. Caron and T. Khan, Aerospace Science and Technology 3, 1999 (513). https://doi.org/10.1016/ S1270-9638(99)00108-X.

20. M. Sundararaman, Mineral Processing and Extractive Metallurgy Review 22, 2001 (681). https:// doi.org/10.1080/08827509808962521.

21. K. Banerjee, Materials Sciences and Applications 2, 2011 (1243). https://doi.org/10.4236/ msa.2011.29168.

22. M. R. Wootton and N. Birks, Corrosion Science 15, 1975 (1). https://doi.org/10.1016/S0010 $-938 X(75) 80024-2$.

23. R. E. Lobnig, H. J. Grabke, H. P. Schmidt and K. Hennessen, Oxidation of Metals 39, 1993 (353). https://doi.org/10.1007/bf00664661.

24. Assessment of surface texture: guidance and general information, BS1134:2010.

25. L. L. C. Minitab, Analysing data, Getting Started with Minitab 18, 23 (2017).

26. "INCA". Oxford Instruments (2014).

Publisher's Note Springer Nature remains neutral with regard to jurisdictional claims in published maps and institutional affiliations. 